\title{
Radius and mass of a transiting $M$ dwarf near the hydrogen-burning limit
}

\section{OGLE-TR-123 ${ }^{\star}$}

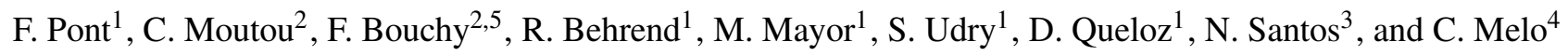 \\ 1 Observatoire de Genève, 51 Chemin des Maillettes, 1290 Sauverny, Switerland \\ e-mail: frederic.pont@obs. unige.ch \\ 2 LAM, Traverse du Siphon, BP 8, Les Trois Lucs, 13376 Marseille Cedex 12, France \\ 3 Centro de Astronomia e Astrofísica da Universidade de Lisboa, 1349-018 Lisboa, Portugal \\ ${ }^{4}$ European Southern Observatory, Casilla 19001, Santiago 19, Chile \\ 5 Observatoire de Haute-Provence, 04870 St-Michel l'Observatoire, France
}

Received 24 June 2005 / Accepted 5 October 2005

\section{ABSTRACT}

The system OGLE-TR-123, detected by the OGLE planetary transit survey in Carina, was shown by subsequent spectroscopic follow-up to be an eclipsing binary with a very low mass companion $\left(M_{2} \sim 0.07 M_{\odot}\right)$. Neither the light curve nor the radial velocity data were sufficient to constrain the parameters more precisely and to exclude alternative explanations in terms of a triple system. In this paper, new HARPS radial velocity data and a precise photometric monitoring of the transit with VLT/FORS2 are presented, resulting in an improved mass and radius determination for OGLE-TR-123b and the rejection of alternative scenarios. We find $M_{2}=0.085 \pm 0.011 M_{\odot}$ and $R_{2}=0.133 \pm 0.009 R_{\odot}$ for the transiting companion, confirming its nature as a small $\mathrm{M}$ dwarf near the hydrogen-burning limit. Its mass-radius relation corresponds to expectations for a relatively young object. The primary is an $\mathrm{F}$ dwarf that does not rotate synchronously with the orbital motion. Asynchronous rotation is unexpected in such a close binary $(P=1.8$ days) but could be explained by the small mass of the secondary, the young age for the system, and the absence of convective envelope in the primary. OGLE-TR-123b is one of only two known objects below $0.1 M_{\odot}$ with directly determined radii, after a similar object was characterised around OGLE-TR-122. The characterisation of OGLE-TR-123 provides another example of the type of system that can closely mimic planetary transits and contaminate transit surveys.

Key words. binaries: eclipsing - stars: low-mass, brown dwarfs

\section{Introduction}

The OGLE planetary transit survey, monitoring Galactic fields since 2001 with the $1.3 \mathrm{~m}$ Warsaw Telescope at Las Campanas (Chile), has identified more that one hundred transiting candidates (Udalski et al. 2002a,b,c, 2003), leading to the detection of five transiting exoplanets (Konacki et al. 2003; Bouchy et al. 2004; Pont et al. 2004; Konacki et al. 2005). The rest of the candidates are eclipsing binaries. As a by-product of the transiting planet search, Bouchy et al. (2005) and Pont et al. (2005a) characterise several dozens of these eclipsing binaries, the majority of them consisting of an $\mathrm{F} / \mathrm{G} / \mathrm{K}$ primary and a small $\mathrm{M}$ secondary, thereby providing direct mass and radius determinations for M dwarfs of all masses. In particular, OGLE-TR-122b (Pont et al. 2005b), with $R_{2}=0.114 R_{\odot}$ and $M_{2}=0.085$ $M_{\odot}$, turned out to be the smallest star so far with a directly

* Based on observations made at ESO (Chile) with HARPS on the $3.6 \mathrm{~m}$ telescope (programme ID 072.C-0488) and with VLT/FORS2 (programme ID 274.C-5052). measured radius. Such objects provide empirical constraints on the mass-radius relationship for stars below $0.1 M_{\odot}$, where models predict that the equation of state evolves from perfect gas to electron-pressure degeneracy.

Another possible diminutive companion close to the hydrogen-burning limit was identified by Pont et al. (2005a) for OGLE-TR-123. This tentative interpretation was based on three radial velocity measurements that show a variation compatible with the transit signal at $P=1.8$ days. However, with only three measurements, more complicated scenarios involving a triple system could not be excluded. Also, the transiting signal found by the OGLE photometry is very shallow ( $~ 8 \mathrm{mmag}$ ), preventing a good determination of the transit shape. Therefore, new spectroscopic and photometric observations were necessary to determine the mass and radius of the transiting companion of OGLE-TR-123. In this paper we present these observations and the resulting parameters for the system. 
Table 1. Radial velocity measurements (in the barycentric frame) and CCF parameters. The first 3 measurements were made with FLAMES (Pont et al. 2005a), and the last 4 with HARPS.

\begin{tabular}{rrrrrr}
\hline \hline $\begin{array}{r}\text { BJD } \\
\mathrm{d}\end{array}$ & $\begin{array}{r}R V \\
\mathrm{~km} \mathrm{~s}^{-1}\end{array}$ & $\begin{array}{r}\text { depth } \\
\%\end{array}$ & $\begin{array}{r}F W H M \\
\mathrm{~km} \mathrm{~s}^{-1}\end{array}$ & $S N R$ & $\begin{array}{r}\sigma_{R V} \\
\mathrm{~km} \mathrm{~s}^{-1}\end{array}$ \\
\hline 2453081.68998 & 0.234 & 5.97 & 44.9 & 10.7 & 0.317 \\
2453084.57979 & -6.208 & 6.13 & 43.4 & 15.2 & 0.215 \\
2453085.67893 & 12.276 & 6.14 & 43.9 & 11.9 & 0.274 \\
2453371.84388 & -8.874 & 5.10 & 51.0 & 3.97 & 1.050 \\
2453406.79455 & 12.447 & 6.23 & 43.3 & 6.15 & 0.515 \\
2453408.77633 & 10.371 & 6.17 & 45.8 & 4.89 & 0.673 \\
2453409.82831 & -7.139 & 6.65 & 45.5 & 3.95 & 0.770 \\
\hline
\end{tabular}

\section{Observations and reductions}

\subsection{Spectroscopy}

The OGLE-TR-123 system was observed in spectroscopy with both VLT/FLAMES at the European Southern Observatory (ESO) Paranal (Chile) and HARPS on the $3.6 \mathrm{~m}$ telescope at ESO La Silla. The FLAMES data was described in Pont et al. (2005a, programme ID 72.C-0191). Four high-resolution echelle spectra were obtained with HARPS in January and February 2005 (programme ID 72.C-0488). Exposure times of one hour per data point were used. Radial velocities and crosscorrelation function parameters for all measurements are given in Table 1. The photon noise is larger for HARPS, due to the telescope size, but still small compared to the amplitude of the observed variation. A rotation velocity of $34 \pm 3 \mathrm{~km} \mathrm{~s}^{-1}$ was inferred in Pont et al. (2005a) from the rotation broadening of the spectral lines, and the HARPS spectra confirm this value.

\subsection{Photometry}

The OGLE-TR-123 system was observed for 6 hours around the expected transit time with the FORS2 camera on the VLT (programme ID 274.C-5052). A continuous sequence of 195 exposures of $15 \mathrm{~s}$ in the $R$ filter was acquired, and four images also taken in the $V$ filter. The observing conditions were good, with seeing decreasing from 0.8 to 0.5 arcsec during the observation sequence. Figure 1 shows the best-seeing image and reveals the presence of a small companion $\sim 1$ arcsec to the northeast of OGLE-TR-123. The companion is 2.5 mag fainter than the main target, thus representing about a tenth of the total flux.

Astrometry was carried out on the FORS2 field based on the USNO Second CCD Astrometric Catalog (UCAC2, Zacharias et al. 2004) with the help of wider images collected on the Swiss Euler $1.2 \mathrm{~m}$ telescope at La Silla. The coordinates of the bright object are $\alpha=11 \mathrm{~h} 06 \mathrm{~m} 51.092 \mathrm{~s}$, $\delta=-61^{\circ} 11^{\prime} 10.68^{\prime \prime}$. The coordinates of the small companion are $\alpha=11 \mathrm{~h} 06 \mathrm{~m} 51.185 \mathrm{~s}, \delta=-61^{\circ} 11^{\prime} 09.79^{\prime \prime}$. A comparison of the $R$ and $V$ images gives $(V-R)=0.78 \pm 0.12$ for the bright object.

The photometric series were reduced with the imagesubtraction software of Alard \& Lupton (1998) and Alard (2000). A PSF-fitting reduction was also tried, but was found to be less efficient, probably due to the small nearby

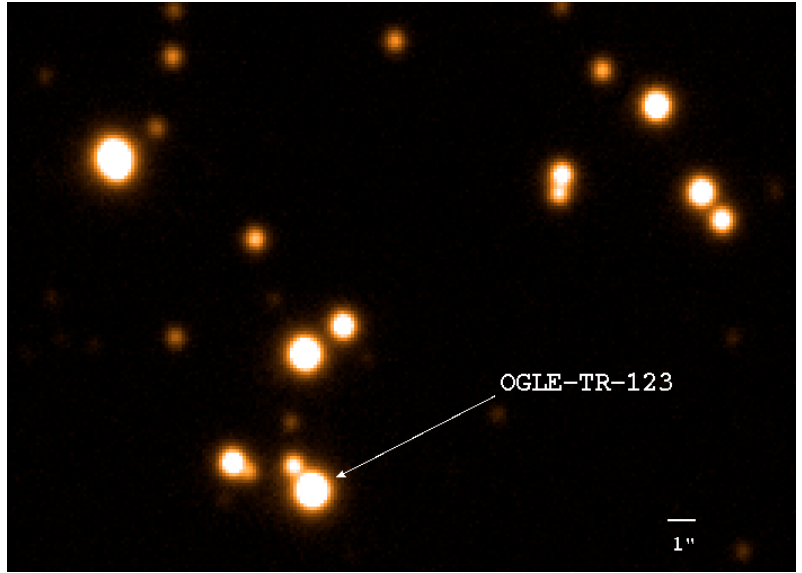

Fig. 1. Detail of a VLT/FORS2 image from our sequence (the image with the best seeing conditions, $\sim 0.5^{\prime \prime}$ ), showing the excellent quality and sampling of the images. North is up and east is left. A faint companion is found about 1 " northeast of OGLE-TR-123.

companion. The subtracted images show that the transit signal is due to the brightest component, not the small companion. The lightcurve is shown in Fig. 4. A very clear transit signal is visible, with much smaller noise than in the original OGLE data (insert in Fig. 4). The photon noise on individual points is around 1.2 mmag. Systematic trends are also visible at the mmag level, especially near the end of the sequence when the seeing was varying more rapidly, but these do not have a significant impact on the derived transit parameters.

\section{Analysis and results}

\subsection{Spectroscopy}

The radial velocity measurements indicate a circular orbit with the period and phase of the transit signal. The orbital period was refined by combining the period obtained by fitting a circular orbit to the radial velocity data, and the period obtained by phasing the transit epoch measured with FORS2 with the original OGLE light curve. We found $P=1.803865$ with an uncertainty of one on the last digit. Fitting a circular Keplerian orbit of this period by least-squares gives $K=11.967 \pm 0.280 \mathrm{~km} \mathrm{~s}^{-1}$ for the semi-major axis (Fig. 2 and Table 2). This solution confirms the tentative orbit derived from the three FLAMES points in Pont et al. (2005b). The O-C residuals are compatible with the observational uncertainties. No second set of lines was observed in the spectra, either from the transiting companion or from a blended contaminant star.

The spectral cross-correlation function (CCF) moves by a significant fraction of its width (about one half) without significant change of shape, which excludes the possibility that the variation is caused by a blended background component. We checked the behaviour of the CCF bisector with time, and found no correlation between the bisector shape and the radial velocity fluctuations. These elements, together with the precise agreements of the derived velocities with a circular orbit, indicate that the Doppler shifts measured in spectroscopy are induced neither by a blend of two line systems nor by the magnetic activity of the primary stars. 


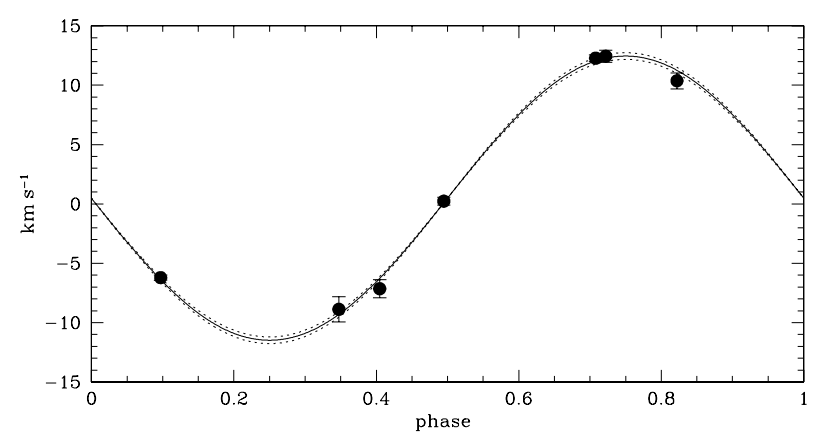

Fig. 2. Phase-folded radial-velocity measurements of OGLE-TR-123, obtained with FLAMES and HARPS, with a fitted circular orbit curve of period 1.803865 day, phased to coincide with the transit events. The dashed lines show the 1-sigma interval on the fitted orbital amplitude.

Table 2. Parameters of the OGLE-TR-123 system (the primary mass and radius are estimated with the help of stellar evolution models).

\begin{tabular}{ll}
\hline \hline Period [days] & $1.803865 \pm 0.000001$ \\
Transit epoch [BJD] & $2453459.6096 \pm 0.0005$ \\
Impact parameter & $0 \pm 0.27$ \\
Radius ratio & $0.0856 \pm 0.0014$ \\
Transit duration [days] & $0.1279 \pm 0.0014$ \\
Eccentricity (fixed) & 0.0 \\
Inclination angle [degrees] & $86-90$ \\
Temperature of the primary [K] & $6700 \pm 300$ \\
Rotation velocity [km s$\left.{ }^{-1}\right]$ & $34 \pm 3$ \\
Systemic velocity $\left[\mathrm{km} \mathrm{s}^{-1}\right]$ & $0.487 \pm 0.204$ \\
Orbital semi-amplitude $\left[\mathrm{km} \mathrm{s}^{-1}\right]$ & $11.967 \pm 0.280$ \\
Mass function & $(0.3210 \pm 0.0225) \times 10^{-3}$ \\
Residuals O-C [km s$\left.{ }^{-1}\right]$ & 0.523 \\
Semi-major axis [AU] & $0.031 \pm 0.002$ \\
& \\
Distance & $1600 \pm 400 \mathrm{pc}$ \\
& \\
Primary mass & $1.29 \pm 0.26 M_{\odot}$ \\
Primary radius & $1.55 \pm 0.10 R_{\odot}$ \\
& \\
Secondary mass & $0.085 \pm 0.011 M_{\odot}$ \\
Secondary radius & $0.133 \pm 0.009 R_{\odot}$ \\
\hline
\end{tabular}

A comparison of the co-added FLAMES spectra with a library of Kurucz stellar spectra allows crude determination of the primary effective temperature (the HARPS spectra have too low a signal-to-noise ratio for this purpose). Figure 3 shows a portion of the stellar spectrum with a comparison of the best-fit library spectra. The derived $T_{\text {eff }}$ is $6700 \pm 300 \mathrm{~K}$. The linebroadening due to rapid rotation prevents a more precise determination of the spectroscopic parameters. The surface of the $\mathrm{CCF}$ is normal for an F star, so that the object cannot be very metal-deficient.

It is noteworthy that the coordinates given by Udalski et al. (2003) for OGLE-TR-123 are nearer to those that we obtained for the small companion than to that of the brightest component. Both HARPS and UVES/FLAMES are fiber-fed spectrographs, with a fiber diameter of 1.0 arcsec on the sky. However, FLAMES uses a fixed pointing, whereas HARPS uses an automatic guiding system that centres the fiber on the brightest target. This implies that the FLAMES spectra - obtained through

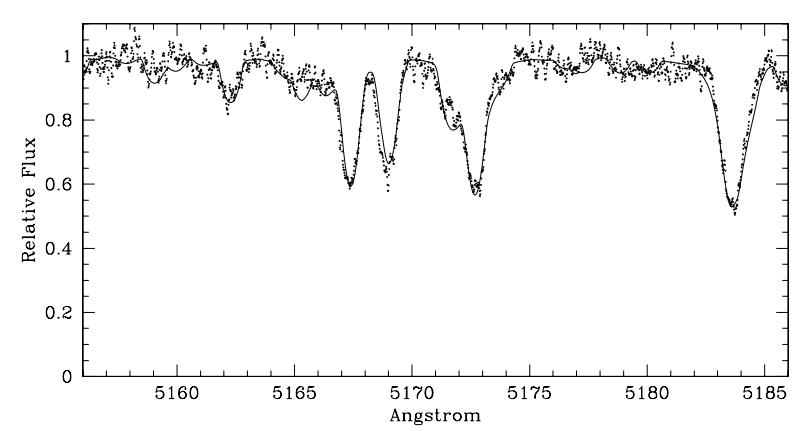

Fig. 3. The primary effective temperature is estimated from the comparison of the FLAMES spectrum with theoretical spectra from Kurucz (2005). Here the data (dots) are compared with a $6700 \mathrm{~K}$ synthetic (line) spectrum in the vicinity of the $\mathrm{MgI}$ triplet.
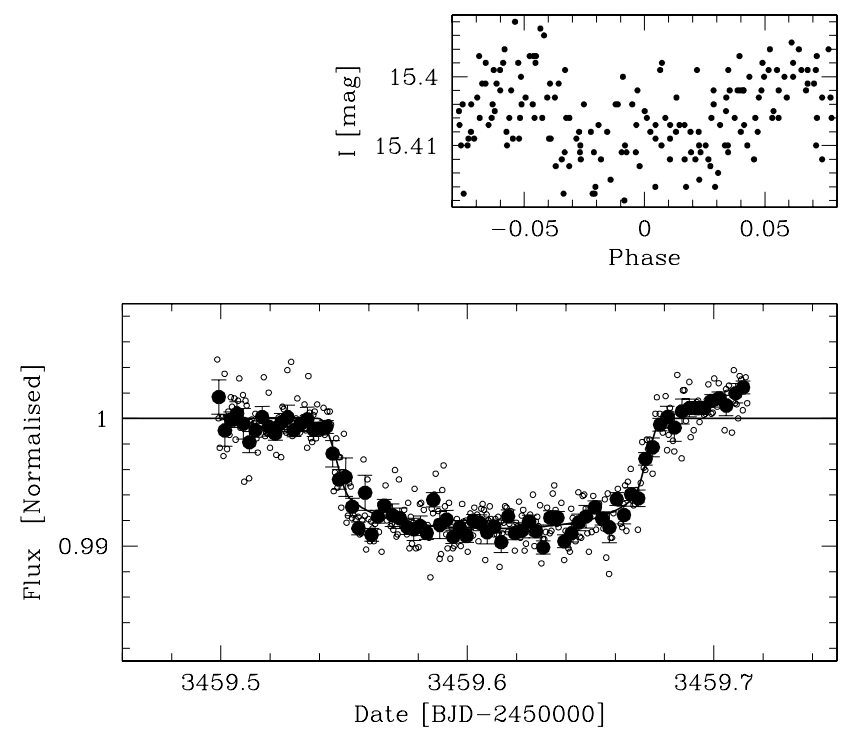

Fig. 4. The FORS2 light curve for OGLE-TR-123, with the bestfitting transit curve. Open symbols show the individual measurements, closed symbols the 5-point averages. The top plot shows the original phase-folded OGLE lightcurve on the same scale.

a fiber placed according to the OGLE coordinates close to the small companion - may have been more contaminated by the companion than was the HARPS spectra. However, the very close agreement of the two sets of radial velocities on a sinusoidal orbit indicate that the effect of the contamination on the radial velocity is negligible. No contribution by a second object is seen in the CCF of either sets of spectra. Simulations confirm that an undetected blended component in the spectra would affect the derived velocity semi-amplitude by a negligible amount.

\subsection{Light curve}

The transit shape was determined from the light curve using the procedure described in Bouchy et al. (2005). The best-fit transit curve is shown in Fig. 4. Realistic uncertainties were determined by taking the systematic trends in the photometry into account in a manner similar to the "residual permutation" method described in Bouchy et al. (2005). A set of synthetic light curves were produced by shifting the residuals around the 
best-fit transit curve by $n$ positions, where $n$ was varied between zero and the total number of exposures. The residuals were shifted in a "circular" manner; i.e. when shifted past the last point in the sequence they were brought back to the first point. The transit fit was repeated on all these curves, and the dispersion in the resulting parameters was used as an indicator of the uncertainties. This method is analogous to the wellknown bootstrap methods, except that it conserves the correlation structure of the residuals, and is therefore better adapted to data with significant systematic trends.

The resulting transit parameters are $T_{0}=2453459.6096 \pm$ $0.0005, b=0 \pm 0.27, R_{2} / R_{1}=0.0856 \pm 0.0007$, and $V_{T} / R_{1}=$ $15.64 \pm 0.17 R_{\odot}$ days $^{-1}$, for the transit central epoch, impact parameter, radius ratio, and transverse velocity, respectively. The FORS2 data therefore indicates a clearly central transit ( $b \ll 1)$. It confirms the values found in Pont et al. (2005a) from the OGLE lightcurve, with much smaller uncertainties. The very short duration of the ingress and egress phases also confirms that the transiting body is much smaller than the primary, therefore excluding a large dilution of the transit by an unrecognised blended star.

The gravitational pull of a massive companion induces a sinusoidal modulation in the light curve of close eclipsing binaries outside the transits, because of the ellipsoidal deformation of the primary (Drake 2003). In the original OGLE light curve for OGLE-TR-123, we found an amplitude of $1.3 \pm 0.6 \mathrm{mmag}$ for this modulation, using the method of Sirko \& Paczyński (2003).

\subsection{Synthesis and system parameters}

The radius ratio and mass function of the system were measured very precisely from the transit signal and radial velocity orbit. The accuracy of the final parameters was limited by the determination of the mass and radius of the primary. Given the short period of the system, assuming synchronous rotation seemed reasonable, but this hypothesis does not produce a meaningful solution. Assuming synchronous rotation, the measured rotation of $34 \pm 3 \mathrm{~km} \mathrm{~s}^{-1}$ implies a primary radius of $R=1.21 \pm 0.04 R_{\odot}$. The transit duration would require $M \leq 0.9 M_{\odot}$ for the primary mass to explain the transit duration (proportional to $\left.R \cdot(m+M)^{-1 / 3}\right)$. Stellar evolution models indicate that such a star would have a temperature lower than $5300 \mathrm{~K}$, in contradiction with the measured temperature indicating an early $\mathrm{F}$ star. Moreover, only model isochrones with ages higher than 14 Gyr satisfy these mass and radius conditions. Such ages are too large for an object belonging to the Galactic thin disc. In our solution we therefore did not use the hypothesis of synchronous rotation. This point is discussed further in Sect. 4.

The parameters from spectroscopy and photometry were combined as described in Pont et al. (2005a). The mass and radius of the primary were derived by combining the constraints from the temperature determined from the spectra, the transit duration and shape, and the stellar evolution models of Girardi et al. (2002), with a global $\chi^{2}$ minimisation (Fig. 5). The mass of the secondary is then obtained from the radial velocity orbit,

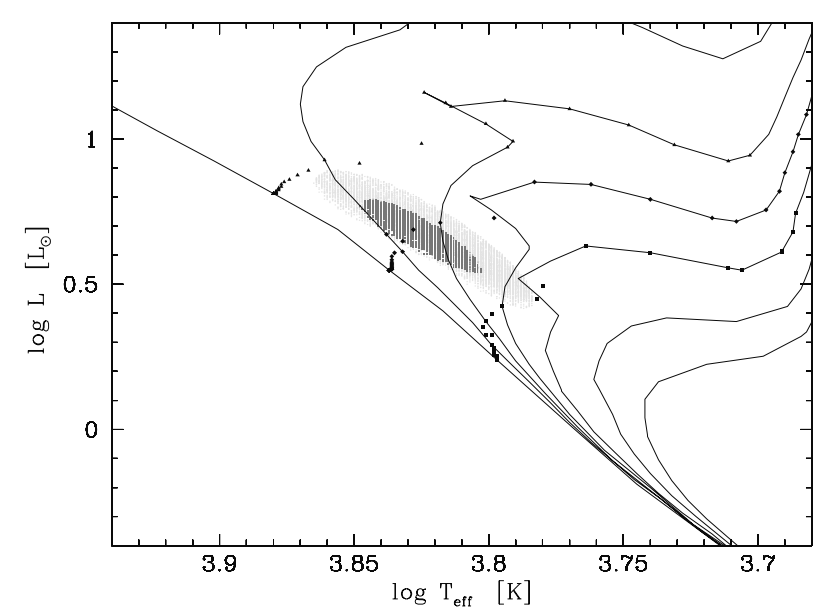

Fig. 5. Position in the temperature-luminosity diagram of the stellar evolution models satisfying the constraints on the primary at the 1-sigma (dark grey) and 2-sigma level (light grey). The constraints come from the spectroscopic temperature and the duration and shape of the transit signal. Theoretical isochrones for solar metallicity and ages $0,1,2,3,5,10$, and $15 \mathrm{Gyr}$ are indicated for reference, evolution tracks for $M=1.2 M_{\odot}$ (squares), $M=1.4 M_{\odot}$, (diamonds) and $M=1.6 M_{\odot}$ (triangles). Models from Girardi et al. (2002).

and its radius from the transit depth and impact parameter. The results are shown in Table 2 . We found $R_{2}=0.133 \pm 0.009 R_{\odot}$ and $M_{2}=0.085 \pm 0.011 M_{\odot}$ for the transiting companion.

The uncertainty on the temperature of the primary permits a range of mass and radius for the primary, which limits the accuracy of the mass and radius of the secondary. A more precise determination of the spectral parameters of the primary would be useful but difficult to achieve, given the faintness and rapid rotation of the target.

A distance estimate was obtained by using the $(V-R)$ colour measured with the FORS2 images, $I=15.40$ from the OGLE photometry, and $M_{I}=2.7 \pm 0.4,(V-R)_{0}=0.23 \pm 0.06$ from the fit of the stellar evolution models. We obtained a distance of $1600 \pm 400 \mathrm{pc}$, with an absorption in $I$ of $a_{I}=$ $1.7 \pm 0.3 \mathrm{mag}$.

\section{Discussion}

The radial velocity and transit data reveal the presence of a small transiting companion around OGLE-TR-123, with a mass near the hydrogen-burning limit and a radius comparable to that of an exoplanet - the second such object characterised by our follow-up of the OGLE transit survey (after OGLE-TR-122b, see Pont et al. 2005b).

The position of OGLE-TR-123b in the mass-radius diagram can be compared to the predictions of the Baraffe et al. (1998) models. For very low-mass M dwarfs and brown dwarfs, the models predict a rapid decrease in size in the first few hundred million years, converging towards a radius that is comparable to Jupiter's. Because of the age dependence, the OGLE-TR-123 system cannot be used to put constraints on the model unless its precise age is known. The models suggest an age between 100 and 500 Myr. Such a relatively young age is also compatible with the early spectral type of the primary 
and with the fact that its rotation appears asynchronous. Tidal effects tend to synchronise the rotation of close binaries with their orbital period. The timescale of this process was studied by Zahn $(1977,1989)$. The synchronisation timescale is found to crucially depend on whether the star's envelope is convective or radiative. The limit between stars with convective and radiative envelopes is thought to be near $1.2 M_{\odot}$. Zahn's formula for stars with a convective envelope predicts a synchronisation timescale of about $10 \mathrm{Myr}$ for the OGLE-TR-123 system. For radiative envelopes, the timescale can be much longer as well as more difficult to determine. Empirically, systems with $a / R<7-8$ are observed to be synchronised (Giuricin et al. 1985). The OGLE-TR-123 system has $a / R \simeq 4.5$. Therefore, even with a radiative envelope, that it does not rotate synchronously is surprising. The fact that the rotation velocity is high could be an indication that the synchronisation process is under way.

The characterisation of the OGLE-TR-123 system also has implications in the context of planetary transit searches. As the second instance after OGLE-TR-122b of a planetary-sized transiting companion, it confirms the importance of planetsized stellar transiting companions as sources of confusion for planetary transit searches. In a case like OGLE-TR-123, the gravitational modulation in the light curve outside the transit (1.3 mmag) could reveal the stellar nature of the companion assuming sufficiently extensive and stable out-of-transit photometry. In our follow-up of the OGLE candidates in Carina, we found two transits by planet-sized stars and three planets, with comparable periods and sizes. This shows empirically that both configurations are found in comparable numbers and that the photometric transit signal alone is not sufficient to establish the planetary nature of a transiting companion. The spectroscopic follow-up constitutes an essential component of planetary transit surveys. Two Doppler measurements even at low signal-to-noise suffice to rule out such imitations of planetary events.
Acknowledgements. The authors wish to thank ESO for timely allocation of DDT time, Ignasi Ribas and Fred Rasio for useful comments on the tidal synchronisation timescale, and the anonymous referee for the careful revision of the manuscript and thoughtful suggestions.

\section{References}

Alard, C. 2000, A\&AS, 144, 363

Alard, C., \& Lupton, R. H. 1998, ApJ, 503, 325

Baraffe, I., Chabrier, G., Allard, F., \& Hauschildt, P. H. 1998, A\&A, 337,403

Bouchy, F., Pont, F., Santos, N. C., et al. 2004, A\&A, 421, L13

Bouchy, F., Pont, F., Melo, C., et al. 2005, A\&A, 431, 1105

Drake, A. J. 2003, ApJ, 589, 1020

Girardi, L., Bertelli, G., Bressan, A., et al. 2002, A\&A, 391, 195

Giuricin, G., Mardirossian, F., \& Mezzetti, M. 1985, A\&AS, 59, 37

Konacki, M., Torres, G., Jha, S., \& Sasselov, D. D. 2003, Nature, 421, 507

Konacki, M., Torres, G., Sasselov, D. D., \& Jha, S. 2005, ApJ, 624, 372

Kurucz, R. 2005, library of synthetic stellar spectra

Pont, F., Bouchy, F., Queloz, D., et al. 2004, A\&A, 426, L15

Pont, F., Bouchy, F., Melo, C., et al. 2005a, A\&A, 438, 1123

Pont, F., Melo, C. H. F., Bouchy, F., et al. 2005b, A\&A, 433, L21

Sirko, E., \& Paczyński, B. 2003, ApJ, 592, 1217

Udalski, A., Paczynski, B., Zebrun, K., et al. 2002a, Acta Astron., 52, 1

Udalski, A., Szewczyk, O., Zebrun, K., et al. 2002b, Acta Astron., 52, 317

Udalski, A., Zebrun, K., Szymanski, M., et al. 2002c, Acta Astron., 52,115

Udalski, A., Pietrzynski, G., Szymanski, M., et al. 2003, Acta Astron., 53, 133

Zacharias, N., Urban, S. E., Zacharias, M. I., et al. 2004, AJ, 127, 3043

Zahn, J.-P. 1977, A\&A, 57, 383

Zahn, J.-P. 1989, A\&A, 220, 112 